\title{
Inspiring Stars - the IAU inclusive world exhibition
}

\author{
Maria Rosaria D’Antonio ${ }^{1}$, Lina Canas ${ }^{2}$ and Wanda Diaz Merced ${ }^{3}$ \\ ${ }^{1}$ IAU Secretariat \\ email: dantonio@iap.fr \\ ${ }^{2}$ IAU Office for Astronomy Outreach \\ email: lina.canas@nao.ac.jp \\ ${ }^{3}$ IAU Office of Astronomy for Development \\ email: wdm@astro4dev.org
}

\begin{abstract}
Inspiring Stars (IS) is an itinerant international exhibition promoted by the International Astronomical Union (IAU) to disseminate world initiatives addressing inclusion in astronomy at the professional level. It encompasses (but is not limited to) outreach, teaching and professional aspects of astronomy, with the ultimate goal of evolving from a levelling the playing field to egalitarian participation by all. Here we present the work-in-progress, a framework of bridging activities and inclusion in the social context, which may provide Inspiring Stars with the potential to motivate the next generation and to document comparable data on inclusion in astronomy.
\end{abstract}

Keywords. Inclusion, resources, equality, diversity

\section{Introduction}

Inspiring Stars (IS) is an itinerant international exhibition promoted by the International Astronomical Union (IAU) to disseminate, highlight, support, motivate and promote inclusive world initiatives addressing equal participation in (but not limited to) astronomy outreach, teaching, and at a professional level any activity in the field of astronomy. Inspiring Stars is a collaborative experience where resources related to (but not limited to) astronomy education, outreach, research and development were collected from around the world and combined into a unique display. The IAU has the aim that this exhibit should become a joint effort by the community and for the community.

This exhibition is a joint venture by the IAU Secretariat, the IAU Office of Astronomy for Development and the IAU Office for Astronomy Outreach. It is sponsored by the American Astronomical Society. By involving the three offices, the IAU intends to bring about a global mobilization for the sustainable and durable addressing of the main issues related to inclusion. In alignment with the IAU's new Strategic Plan, the exhibition links the three branches together: research, development and communication through the lens of inclusion. IAU national member states will also be asked to actively participate in hosting an exhibition in their country. The Mini-Task Force also collaborates closely with other IAU scientific bodies, such as the IAU Equity and Inclusion Working Group and IAU Commission C1, for Astronomy Education and Development.

The exhibition premiered during the IAU General Assembly 2018 in Vienna, from 20-31 August. Its debut in Austria took place on two occasions: a bridging activity when the team visited Vienna in April 2018 and contacted local organisations, exchanging experiences; and the debut of the exhibition itself at the General Assembly in August when all elements, international and local, were combined. 


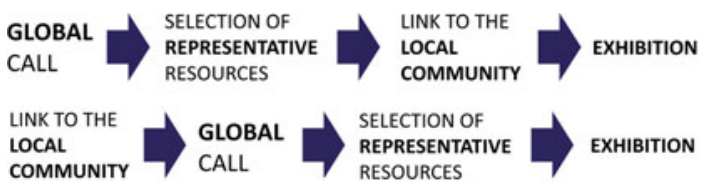

Figure 1. Top: Structure of the first implementation strategy: first issuing a global call, the selection of representative resources, establish a link to the local community and finally the exhibition. Bottom: Structure of the following current implementation strategy: first establish a link to the local community, then issuing a global call for resources, the selection of representative resources and the exhibition (Schematics Credit: IAU/Inspiring Stars). An extra step is predicted for establishing the criteria for selection.

Inclusive both in goals and practices, the Inspiring Stars exhibition changes and grows with the communities it visits - assimilating the best practices around the world, and for the community - by incorporating and disseminating different local efforts. By implementing this strategy the project is seeking to:

(a) Establish a larger network of contacts locally (e.g. more geographical representation, more diversity in disabilities and/or impairments);

(b) Involve the local astronomy community;

(c) Strategize educational campaigns for the fostering of local collaborations;

(d) Strategize the emergence of new technologies and initiatives and predict new ones;

(e) Implement strategies for educational campaigns to promote egalitarian participation in all aspects of participation in astronomy (education, outreach and professional activities see Fig. 1);

$(f)$ Establish a number of dedicated grants for inclusion, embracing astronomers from vulnerable countries and those which are in conflict.

The main characteristic being an itinerant exhibition, the key actions being carried out vary, being contextualized according to the needs of the community it is serving - in particular, according to the target audience and designated area for the exhibition. The deliverables predicted for each Exhibition event comprise:

- Two activities implemented for each location: Bridging \& Exhibition;

- Web page (that will include the local partner organization, the programme for the exhibition launch, a list of contents showcased, etc.);

- List of resources identified and showcased;

- Issuing an international Call for Activities/Resources;

- Original Exhibition Panel production (from content drafting to printing).

\section{Framework}

Inspiring Stars has made an inter-connection between four different but equally relevant frames of action surrounding inclusion. In each case, the theme is for inclusion regardless of race, geographical origin, academic background, ethnicity, socio-economic background, sexual orientation, gender identity, disability or religion.

- For Human Development we wish to create opportunities for diverse people, with different performance styles, and with different perspectives, and hence to generate a pool of people with different skills and oversee the growth of mutual understanding;

- For Outreach and Communications we wish to create opportunities for a better relationship between communication and research and to generate a wider diversity and display of interests, a wider reach, better engagement, better access to information and communications skills development;

- For Education we wish to develop new methodologies and to enable access to quality information in the classroom and to help in the creation of teacher support 


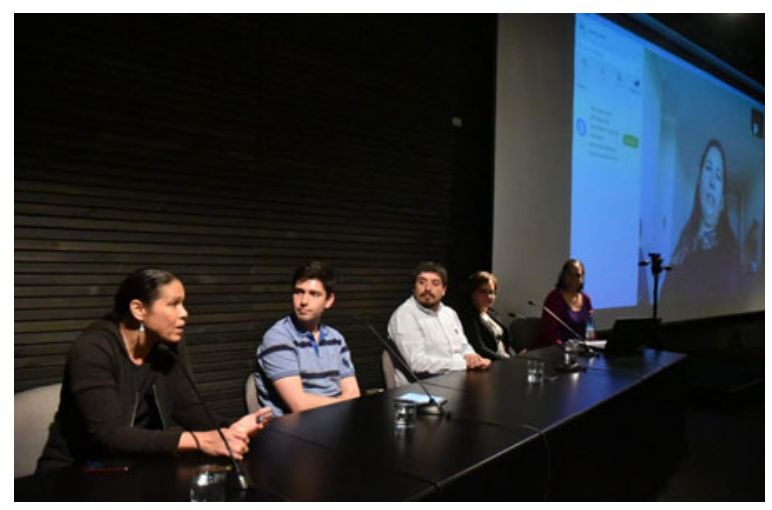

Figure 2. Inspiring Stars was presented in Santiago, Chile highlighting the Chilean community's many efforts on inclusive astronomy through the project "Astronomy for All", a national cooperation of astronomical institutions helping to bring astronomy to everyone in an accessible way. Credit: IAU/Astronomy for All/Erika Labbe.

programmes, with access to new experiences in the classroom and the improvement of digital technologies;

- For Scientific Research we wish to generate for persons with congenital and acquired disabilities and/or other impairments a path that permits more publications, more innovation, more citations, a higher impact factor, and to promote an increase in the presence of such persons, so as to enrich their work in the field of human development, with fairness and progress in the working environment.

\section{Implementation Overview}

The Inspiring Stars project began its preparation for the IAU General Assembly in November 2017, with the attempt of getting the Austrian community of astronomers involved and the Austrian organizations serving people with disabilities and/or impairments. To bring both these groups together, it took the team almost one year.

A bridging event was carried out in April 2018, where local and international astronomers met with individuals of the organizations serving these people with disabilities and/or impairment to talk about making the world of astronomy inclusive in all aspects of participation (i.e. the workforce, university, academia, education from University-level to school, and outreach). The bridging activities so far have been very effective in building trust with the community of those with disabilities and/or impairments and getting the professional community involved. The participants have expressed that it has given them a voice they never realized they had in science and it has strengthen their collaboration. So far, since the inauguration in August, 2018 one bridging event (hosted in Chile, see Fig. 2) has been carried out over a two-month span.

The day of the inauguration at the General Assembly, the Inspiring Stars exhibition opened to receive those organizations of people with disabilities and/or impairments, with participants from schools and astronomers to inaugurate the exhibit. Out of the 3000 astronomers at the general Assembly, we estimate that 500 people came to the exhibit, visited the inclusive stations and listened to the talks during the special opening day dedicated to inclusion. The events comprised panels on Journal Access, presentations on data access and the work from inclusive initiatives which were showcased at the different stations.

The feedback from the organizations participating was very positive and praised highly the opportunity to interact both with the new technologies and with the practising 
community. The organizations nominated Inspiring Stars for an Austrian prize called Youth-Project-Award and to participate in the United Nations Zero project conference to be held in August 2019.

\section{Considerations}

After the first year, now that a first full cycle of implementation has been finalized, the lessons learned from this debut event are now being carefully identified and addressed.

There is a need to focus on establishing a stronger and larger network of contacts locally, including having more geographical linkages and more diversity in the disabilities and/or impairments considered. For this first bridging event, the astronomy community was involved very little, and the project missed reaching the research community at the national level. The project needs to understand what is the percentage of the national astronomical community that actually participates in the exhibition. Regarding on-site measures, it is paramount to establish a proper meeting with all the exhibitors involved, and to stress the importance of having a quiet room reserved at each exhibition venue. Such matters need to be in the draft guidelines for event coordinators.

One of the first steps that the project will produce is the IAU Inspiring Stars Endorsement criteria for inclusive astronomy resources and/or activities. The IAU Inspiring Stars is setting up a mechanism to endorse inclusive astronomy resources and/or activities. A process is to be set in place that establishes transparent mechanisms and criteria for endorsement.

Although there is always room for improvement, the impact is already showing: as a result of the bridging activity in Chile, the Universidad Diego Portales is dedicating a funding allocation to research on journal access, the American Astronomical Society will assemble the exhibition and mobilize astronomers from the US. People are responding and reaching out to the Office of Astronomy for Development in order to learn how to work on inclusion, especially in relation to research. The Office of Astronomy for Outreach submitted a proposal to study ways to effectively integrate inclusion into its current networks, so as to support and disseminate all aspects of information to the amateur astronomy and public outreach communities, and ultimately to make it easier for anyone to access information about our Universe. In a year of research, they will study and address the barriers and methods to promote the systematic application of research findings on inclusive accessibility in astronomy outreach, education and professional research activities.

Regarding impact, our challenge is to get the professional community to attend to these activities and to change their mindset on inclusion being not directly related to them and that it has no impact on their research projects. Inspiring Stars will add the focus of streamlining all aspects of participation through educational campaigns. In that respect, in five years we hope that the development of a mathematical technique to analyse astronomy information is published in high impact journals, and equally, if a sensory technique is developed to analyse astronomical data and this is mathematically sustainable, then it too has to be published. On the other hand, there is still a lot of work to do, especially regarding changing the mindsets of individuals and to promote active participation in the mainstream community, and we cannot underestimate the efforts that achieving this will take. 